\title{
Comparison of machine learning and deep learning techniques in promoter prediction across diverse species
}

\author{
Nikita Bhandari ${ }^{1}$, Satyajeet Khare ${ }^{\text {Corresp., } 2}$, Rahee Walambe ${ }^{\text {Corresp., } 3,4}$, Ketan Kotecha ${ }^{1,3}$ \\ ${ }^{1}$ Computer Science, Symbiosis Institute of Technology, Symbiosis International (Deemed University), Pune, MH, India \\ 2 Symbiosis School of Biological Sciences, Symbiosis International (Deemed University), Pune, MH, India \\ 3 Symbiosis Centre for Applied Artificial Intelligence, Symbiosis International (Deemed University), Pune, India., Pune, Maharashtra, India \\ 4 Electronics and Telecommunication Dept, Symbiosis Institute of Technology, Pune, Pune, Maharashtra, India \\ Corresponding Authors: Satyajeet Khare, Rahee Walambe \\ Email address: satyajeet.khare@ssbs.edu.in, rahee.walambe@scaai.siu.edu.in
}

Gene promoters are the key DNA regulatory elements positioned around the transcription start sites and are responsible for regulating gene transcription process. Various alignment-based, signal-based and content-based approaches are reported for the prediction of promoters. However, since all promoter sequences do not show explicit features, the prediction performance of these techniques is poor. Therefore, many machine learning and deep learning models have been proposed for promoter prediction. In this work, we studied methods for vector encoding and promoter classification using genome sequences of three distinct higher eukaryotes viz. yeast (Saccharomyces cerevisiae), A. thaliana (plant) and human (Homo sapiens). We compared one-hot vector encoding method with frequency-based tokenization (FBT) for data pre-processing on 1-D Convolutional Neural Network (CNN) model. We found that FBT gives a shorter input dimension reducing the training time without affecting the sensitivity and specificity of classification. We employed the deep learning techniques, mainly CNN and recurrent neural network with Long Short Term Memory (LSTM) and random forest (RF) classifier for promoter classification at k-mer sizes of 2, 4 and 8 . We found CNN to be superior in classification of promoters from non-promoter sequences (binary classification) as well as species-specific classification of promoter sequences (multiclass classification). In summary, the contribution of this work lies in the use of synthetic shuffled negative dataset and frequency-based tokenization for pre-processing. This study provides a comprehensive and generic framework for classification tasks in genomic applications and can be extended to various classification problems. 
1

\section{Comparison of machine learning and deep learning}

3 techniques in promoter prediction across diverse species

4

5 Nikita Bhandari ${ }^{1}$ Satyajeet Khare ${ }^{4}$ Rahee Walambe ${ }^{2,3}$ Ketan Kotecha ${ }^{1,3}$

6

$7{ }^{1}$ Computer Science, Symbiosis Institute of Technology, Symbiosis International (Deemed

8 University), Pune, MH, India

92 Electronics and Telecommunication Dept, Symbiosis Institute of Technology, Pune,

10 Maharashtra, India

$11{ }^{3}$ Symbiosis Center for Applied AI (SCAAI), Symbiosis International Deemed University, Pune,

12 Maharashtra, India

$13{ }^{4}$ Symbiosis School of Biological Sciences, Symbiosis International Deemed University, Pune,

14 Maharashtra, India

15

Corresponding Authors:

17 Satyajeet Khare ${ }^{4}$

18 Symbiosis School of Biological Sciences, Pune, Maharashtra, India, 412115

19

20

21

Rahee Walambe ${ }^{2,3}$

Symbiosis Institute of Technology, Pune, Maharashtra, India, 412115

23

Email address: rahee.walambe@sitpune.edu.in

24

25

26

27 
35

36

37

38

39

40

41

42

\section{Abstract}

Gene promoters are the key DNA regulatory elements positioned around the transcription start sites and are responsible for regulating gene transcription process. Various alignment-based, signal-based and content-based approaches are reported for the prediction of promoters. However, since all promoter sequences do not show explicit features, the prediction performance of these techniques is poor. Therefore, many machine learning and deep learning models have been proposed for promoter prediction. In this work, we studied methods for vector encoding and promoter classification using genome sequences of three distinct higher eukaryotes viz. yeast (Saccharomyces cerevisiae), A. thaliana (plant) and human (Homo sapiens). We compared onehot vector encoding method with frequency-based tokenization (FBT) for data pre-processing on 1-D Convolutional Neural Network (CNN) model. We found that FBT gives a shorter input dimension reducing the training time without affecting the sensitivity and specificity of classification. We employed the deep learning techniques, mainly $\mathrm{CNN}$ and recurrent neural network with Long Short Term Memory (LSTM) and random forest (RF) classifier for promoter classification at k-mer sizes of 2, 4 and 8 . We found $\mathrm{CNN}$ to be superior in classification of promoters from non-promoter sequences (binary classification) as well as species-specific classification of promoter sequences (multiclass classification). In summary, the contribution of this work lies in the use of synthetic shuffled negative dataset and frequency-based tokenization for pre-processing. This study provides a comprehensive and generic framework for classification tasks in genomic applications and can be extended to various classification problems.

Keywords: Promoter Prediction, Deep Learning, Machine Learning, CNN, LSTM, Random Forest, One-hot Encoding, Frequency-based Tokenization

Introduction

Accurate transcription of a gene requires RNA polymerase enzyme to recognize the start site of the gene and the end. One of the key regions involved in the transcriptional regulation of RNA present at the start site is called promotor. A fundamental requirement for establishment of gene expression pattern and regulatory network is enabled by promoters. The selection of promoter is an important factor for Genetic engineering. It is used to manipulate gene architecture and expression of genes under various conditions (Singla-Pareek, Reddy, and Sopory 2001). Promoter sequences have a gene-specific architecture which makes it hard to identify them computationally. A strong TATA box is present in a number of promoters of highly expressed genes. On the other hand, multiple groups of genes manifest into the TATA-less promoters. In the last decade, genomes of several organisms have been sequenced. Though the gene information has been computationally recognized, the size and functional features of the 
promoters are still left largely undetermined in newly sequenced genomes (Umarov and Solovyev 2017).

Prediction of promoters can be achieved in various ways e.g. simple sequence alignment, content-based approach, or signal-based approach, etc. Matching gapped fingerprints of unlabelled sequences with labelled sequences is an example of a sequence alignment approach (Gordon et al. 2003; J. T. L. Wang et al. 1999). The signal-based approach considers promoter elements such as TATA box, CCAAT-box, etc. as signals for prediction and ignores the nonelement portion of the sequence resulting in poor prediction performance (Knudsen 1999). The content-based approach considers the frequency distribution of k-mer fragments, for example, considering region with a high frequency of CpG sites (Ioshikhes and Zhang 2000; Y. Li, Chen, and Wasserman 2016). CpGProD (Ponger and Mouchiroud 2002), McPromoter (Ohler 2000), CONPRO (Liu and States 2002), Eponine (Down and Hubbard 2002), FirstEF(Davuluri 2003) are some examples of signal-based and content-based approaches used for eukaryotic promoter prediction. The tuning of sequence alignment completely relies on the systematic usage of reference sequences. This can degrade the performance of distantly related set of sequences (Mathur 2013). Also, sequence alignment methods are relatively less effective due to their heuristic nature and high memory requirement for alignment of longer sequences (Chowdhury and Garai 2017; Gordon et al. 2003). ConSite (Sandelin, Wasserman, and Lenhard 2004), rVISTA (Loots et al. 2002), PromH (Solovyev and Shahmuradov 2003), FootPrinter (Blanchette and Tompa 2003) are sequence alignment based promoter prediction applications.

Machine learning (ML) and deep learning (DL) techniques play an important role in this area as compared to regular statistical methods mentioned above. ML techniques have been used in a variety of applications of genomics such as identification of splice site (Larrañaga et al. 2006; Nguyen et al. 2016), promoters regions (Anwar et al. 2008; Lai et al. 2019; Rahman et al. 2019), classification of diseased related genes (Díaz-Uriarte and Alvarez de Andrés 2006; Le Thi, Nguyen, and Ouchani 2008), identification of transcription start site (TSS)(Libbrecht and Noble 2015), identification of protein binding sites (Pan and Yan 2017), recognition of genomic signals such as polyadenylation sites and translation initiation sites (Kalkatawi et al. 2019), disease diagnosis (Manogaran et al. 2018), transcriptomics analysis (Karthik and Sudha 2018), drug discovery and repurposing (Cheng et al. 2019), identification of biomarkers (Tabl et al. 2019),etc. Though the applications of ML and DL techniques in the field of genomics are growing, accurate prediction of promoters is still one of the most challenging tasks in genomics (Anwar et al. 2008; Oubounyt et al. 2019; Umarov and Solovyev 2017).

Various attempts of application of ML and DL algorithms have been made in promoter prediction. E.coli promoters provide a simple model system to study the promoter prediction. Specifically, $\sigma 70$ promoters from E.coli were subjected to intense investigation in the following studies. In one of the studies, 669 promoter sequences, each with length of 80 nucleotides were analysed using a synthetic background and a feedforward neural network with three layers resulting in 96\% precision(Rani, Bhavani, and Bapi 2007). In another study, an ensemble of 
109

110

111

112

113

114

115

116

117

118

119

120

121

122

123

124

125

126

127

128

129

130

131

132

133

134

135

136

137

138

139

140

141

142

143

144

145

146

Support Vector Machine, Linear Discriminant Analysis and Logistic regression was utilized which resulted in a classification accuracy of $86.32 \%$ for promoter sequences (Rahman et al. 2019). One of the studies has used 106 records of $\sigma 70$ promoter and non-promoter sequences to train the model using CNN. For vectorization of input sequences, they applied one hot encoding with the k-mer size of 3 nucleotides. This study came up with an accuracy of $99 \%$ (Nguyen et al. 2016). However, the smaller size of the dataset for deep learning remains a concern. A promoter predictive model based on CNN(PPCNN) achieved much better sensitivity and specificity on E.coli $\sigma 70$, Arabidopsis and human promoters (Umarov and Solovyev 2017). However, the sample size of the promoter and non-promoter sequences was variable for these organisms (81 base pairs for E.coli $\sigma 70$ promoter whereas 251 base pairs for other organisms). Another approach employed SVM to discriminate promoters and non-promoters of five different organisms (plants (various species), Drosophila, Homo sapiens, Mus musculus, Rattus norvegicus) using k-mer size 4 (Anwar et al. 2008). With the test set of 100 sequences (50 promoter and 50 non-promoter sequences) for each species, an average accuracy of $88 \%$, sensitivity of $86 \%$ and specificity of $87.6 \%$ was achieved.

Considering the previous work in the area of sequence classification into promoter and nonpromoter categories using ML and DL methods, there is scope for further improvements in terms of prediction performance. Factors such as sequence length, k-mer size, selection of negative dataset, feature encoding technique will help to achieve the accurate prediction of promoter sequences. Also, the highly imbalanced positive and negative sample dataset is one of the major problems in promoter recognition as it leads to model overfitting and makes the model less generic. Additionally, randomly selecting a non-promoter region from the same genome as negative datasets has its own limitation as the training model tends to find very simple features. Several studies have used one-hot encoding for the sequence encoding process(Giosue and Gangi 2017; Lai et al. 2019; Nguyen et al. 2016; Oubounyt et al. 2019; Rahman et al. 2019).

The objective of this study is to overcome the limitations listed above and to make a classifier more robust and generic. We collected the promoter and non-promoter sequences of 3 distinct organisms: Yeast, A. thaliana, Human and developed an effective and powerful promoter classifier using deep convolutional neural network. We have also used the frequency-based tokenization approach instead of one-hot encoding for feature vectorization for various k-mer sizes (2-mer, 4-mer, 8-mer). The classifier successfully distinguished promoter from nonpromoter sequences with very high sensitivity, specificity, and accuracy. The same CNN model was used for the cross-species evaluation and multi-species classification of promoter sequences. CNN showed high efficiency in promoter prediction when compared with the LSTM and RF classifier. This technique can be extended automatically for the recognition of complex functional elements in sequence data from biological molecules.

\section{Methods}

\section{Selection of Dataset}


147

148

149

150

151

152

153

154

155

156

157

158

159

160

161

162

163

164

165

166

167

168

169

170

171

172

173

174

175

176

177

178

179

180

181

182

183

184

We selected yeast, $A$. thaliana and human genomes for our analysis. The genomes were selected using UCSC genome browser (Haeussler et al. 2019). We collected the datasets of approximately 41671 sequences of $A$. thaliana (UCSC version araTha1), 61546 sequences of humans (UCSC version hg38) each, and 6125 sequences of yeast (UCSC version sacCer3). However, after preprocessing and data cleaning, we randomly selected 35000, 35000 and 6000 sequences for A. thaliana, human and yeast respectively for experimentation. One thousand basepairs (bp) long putative promoter regions ( -700 to $+300 \mathrm{bp}$ around TSS) were extracted in two steps as follows: As a first step, 700 bases upstream of the TSS were selected in the Table Browser using RefSeq Genes as an input to create a custom track. As a second step, 300 bases downstream were selected using custom track as an input to extract a 1000 bases promoter sequence in a FASTA format. Equal number of background sequences were generated using a synthetic method of shuffling of promoter sequences for each organism (Caballero et al. 2014). The ratio of positive and negative sequences for each organism was 1:1. Each of these datasets were processed for the development of distinct models. The number of samples were divided into training and testing sets with the split of $90 \%$ and $10 \%$, respectively. Out of $90 \%$ of training samples $10 \%$ of data was considered for validation and parameter tuning. We have utilised stratified random sampling for splitting of data, and therefore equal percentage of samples of each target class were used for training and testing of models. These datasets were further subjected to feature extraction, feature encoding and classification purpose (Fig. 1). Number of learning epochs used were 10 and the batch size was 128 . Binary cross-entropy and sparce categorical cross-entropy were used as loss function for binary and multiclass classification, respectively. Adam optimizer was utilized with default learning rate. Early stopping callbacks was monitored through loss on the validation dataset. The efficiency of the models was also tested using human sequence data. 1000 nucleotides long sequences were extracted from the human genome (hg38) using fastasubsample script of MEME Suite (v 5.0.5) (Bailey et al. 2009). These sequences were used as negative dataset to test the efficiency of the model.

\section{Data Pre-processing}

DNA sequences are 1D-channel of four nucleotides, adenine (A), thymine $(T)$, guanine $(\mathrm{G})$ and cytosine $(\mathrm{C})$. Feature extraction process is used to generate input for ML/DL models from raw data of nucleotide sequences. For feature extraction we used k-merization process. In this process, a short sequence segment of k consecutive nucleotides (k-mers) with the stride of 1 were generated from parent sequences (Chor et al. 2009). We specifically studied the effect of featureextraction parameter k-mer size on the prediction performance of the models. We used four different k-mer sizes for experimentation: 1-mer, 2-mer, 4-mer and 8-mer. Further, k-merized sequences were given as input to the feature encoding techniques.

\section{Feature Encoding}

We used two different approaches for feature encoding viz. frequency-based tokenization and one-hot encoding. In frequency-based tokenization, each k-mer in the sequence is encoded into 
185

186

187

188

189

190

191

192

193

194

195

196

197

198

199

200

201

202

203

204

205

206

207

208

209

210

211

212

213

214

215

216

217

218

219

220

221

tokens based on its frequency and mapped to a unique index starting from 1. Zero is a reserved index not assigned to any k-mer. This helps transform each sequence of k-mers to a sequence of integers. Feature encoding was achieved using in-house script written in Python (version 3) with system specification as: 64bit operating system, x64 based processor, 8GB RAM, intel CORE i7 processor. We used a text tokenization utility class of Keras pre-processing and data augmentation module for feature encoding. Whereas for one-hot encoding each k-mer is represented by a new binary variable. For this we utilized the OneHotEncoder class of Scikitlearn preprocessing utility. The output of Frequency based tokenization and one-hot encoding were subjected to CNN based analysis. The output of frequency-based tokenization was also subjected to other ML and DL analyses viz. random forest and LSTM RNN.

\section{Random Forest Classifier}

Random forest is an ensemble of decision trees constructed using a different sample from the original data (Breiman 2001). First, each tree is built from a random bootstrapped sample of the training data. Second, at each split of the tree, RF model considers only a small subset of features for training. This helps to improve generalization by reducing variance. The final classification is obtained by combining results from the decision trees passed by votes. The bagging strategy of RF can effectively decrease the risk of overfitting when applied to large dimension data. Therefore, RF can handle a large data set with high dimensionality. Random forest has been widely used in the prediction of DNA-binding proteins(L. Wang, Yang, and Yang 2009), microarray data analysis (Yang et al. 2010), regulatory elements prediction (R. Li et al. 2017), etc. The encoded data are given as input to the Random Forest Classifier of scikit-learn ensemble module for classification of promoters and non-promoter sequences (Pedregosa et al. 2011). The value of $n$ _estimator (number of trees in the forest) was 300, minimum number of samples required to split an internal node was 2 and bootstrap value was false.

\section{LSTM-Recurrent Neural Network}

For sequential data, the flow of gradients for long durations can help in learning long-term dependencies. This can be achieved using Long Short-Term Memory(LSTM) RNN (Hochreiter and Schmidhuber 1997). We fed the pre-processed encoded input to the LSTM layer. The output of the LSTM layer with 128 units was fed to a Dense layer and the output of the Dense layer with 64 units was fed to the Drop-out layer and used Relu activation. In the Dropout layer half the units were dropped. The final state was mapped through a fully connected layer with Sigmoid activation. We used LSTM, Activation, Dense, Dropout and Embedding methods from layer module of Keras (version: 2.2.4) to develop this architecture. Performance evaluation parameters are further used to evaluate the performance of the LSTM model.

\section{Convolutional Neural Network}

Encoding helps to represent 1-D channel of DNA sequence in the form of a sequence of numerical values. This form of representation can be used as input to convolutional neural 
222

223

224

225

226

227

228

229

230

231

232

233

234

235

236

237

238

239

240

241

242

243

244

245

246

247

248

249

250

251

252

networks (Collobert and Weston 2008; LeCun et al. 1998). To build CNN architecture, we utilized three 1D convolution layers, each followed by a max-pooling layer. Each convolutional layer uses filter of size 5 . The pool size of the max-pooling layer was 4 with stride 1 . The output of the max-pooling layer was then fed to three fully connected dense layers, consisting of 1025 , 512,128 units and used the ReLu activation function with a $20 \%$ dropout. The final one is the classification layer and uses the sigmoid activation. We utilized Conv1D and MaxPooling1D methods from the layer module of Keras (version: 2.2.4) for experimentation. Performance evaluation parameters are further used to evaluate the performance of the CNN model.

\section{Performance Evaluation Parameters}

After the application of the prediction model on the dataset, we obtained true positive (TP) and true negative (TN) numbers of truly identified promoter and non-promoter sequences. We also obtained false positive (FP) and false-negative (FN) numbers of falsely identified promoter and non-promoter sequences. In order to evaluate the performance of classification models we computed accuracy (Acc), sensitivity (Sn), specificity (Sp) (Skaik 2008) and Matthews correlation coefficient (MCC) (Matthews 1975) using following equations:

$$
\text { Accuracy }=\frac{T P+T N}{T P+T N+F P+F N}
$$

$$
\text { Sensitivity }=\frac{T P}{T P+F N}
$$

$$
\text { Specificity }=\frac{T N}{T N+F P}
$$

$$
M C C=\frac{((T P * T N)-(F P * F N))}{\sqrt{((T P+F P) *(T P+F N) *(T N+F P) *(T N+F N))}}
$$

\section{Results}

\section{Selection of genomic datasets}

We selected three eukaryotic genomes viz. yeast, A. thaliana, and humans, for classification of sequences into the promoter and non-promoter groups. Yeast being the smallest genome, resulted in a small dataset of $\sim 6000$ promoters. Large genomes of $A$. thaliana and humans generated datasets of $\sim 35000$ promoters each. Negative datasets were generated by reshuffling the promoter sequences. The data were divided into train and test sets with a split of $90 \%$ and $10 \%$, respectively. The $10 \%$ of training data was used for validation and parameter tuning. The resulting datasets were used for comparison of feature encoding techniques and training ML/DL models. In order to evaluate the performance of the ML/DL models, a dataset of 600 random sequences from human genome was used. 
253

254

255

256

257

258

259

260

261

262

263

264

265

266

267

268

269

270

271

272

273

274

275

276

277

278

279

280

281

282

283

284

285

286

287

288

289

\section{Comparison of Feature encoding techniques}

We tested efficiency and computational requirement of one-hot encoding and frequency-based tokenization (FBT) techniques. For this purpose, we used yeast dataset and tested the feature encoding methods on k-mer sizes 1 and 2 (Table 1). We found that at both k-mer sizes the training time was higher for one-hot encoding without significant improvement in sensitivity and specificity. For one-hot encoding, the testing accuracy of CNN model was $95 \%$ for 1 -mer and $96 \%$ for 2 -mer whereas for FBT accuracy achieved was $97 \%$ for 1 -mer and $96 \%$ for 2 -mer. We also trained higher k-mer sizes (more than 2-mer) however the training time required was very high at the described configuration. Based on these results and the fact that we wanted to use kmer sizes 2, 4 and 8; we decided to use FBT for further work.

\section{Comparison of ML/DL algorithms in binary classification}

The first set of experiments was conducted to perform binary classification of sequences into the promoter and non-promoter groups. The performance of CNN, LSTM and RF are shown in Table 2 for the yeast, $A$. thaliana, and human datasets. The average performance of the CNN model on the test set of sequences was: Sn-95.33\%, Sp-97.5\%, Acc-96\%, Mcc-92.5\%. It was found that the computed CNN model performed better than the LSTM and RF for all k-mer sizes. For k-mer size 2, RF performed better than LSTM. However, LSTM tends to work significantly better for k-mer sizes 4 and 8 (Fig. 2a, 2b, 2c). Then we analysed the efficiency of these models on each of the three organisms. In all three species, CNN outperformed LSTM and RF in all evaluation metrics (Fig. 2d, 2e, 2f). Both CNN and RF do not show any pattern in change in the accuracy with k-mer size. Accuracy of CNN remains high throughout for all k-mer sizes. Accuracy of LSTM on the other hand improves with increase in k-mer size. However, we have observed that the computational requirement for LSTM increases with increase in k-mer size.

\section{Cross Species evaluation using ML/DL models}

We also performed a cross-species evaluation using CNN and RF. The purpose of the crossspecies evaluation was to test whether sequence structures that underlie promoter regions are conserved across species (Lai et al. 2019). In cross-species evaluation, we trained the CNN and RF models using one organism's data and evaluated on the rest of the organisms for all k-mer sizes considered. We can see that the average accuracy performance of CNN dropped to $72.77 \%$ and that of RF dropped to $55.66 \%$. This result suggests that there is a large variation in sequence structures of promoters across species (Fig. 3).

\section{Testing of ML/DL model with random genome sequences}

Finally, in order to evaluate the performance of these ML/DL models, we also used random sequences from human genome as negative data with testing data. The prediction performance of CNN model was better than LSTM and RF. The average accuracy achieved by CNN model is $91 \%$, along with $88 \%$ sensitivity, $99 \%$ specificity and $85 \%$ Mcc (Table 3 ). 


\section{Comparison of ML/DL algorithms in multiclass-classification}

291 From cross-species evaluation we found that promoters from the different species have different

292

293

294

295

296

297

298

299

300

301

302

303

304

305

306

307

308

309

310

311

312

313

314

315

316

317

318

319

320

321

322

323

324

325

326

327

328 sequence structures. Therefore, we performed the multispecies classification of sequences to a promoter category. We created a sample dataset of $\sim 66000$ promoter sequences from yeast, Arabidopsis thaliana, and human. Multiclass classification performance of CNN, LSTM, and RF models for various k-mer sizes are shown in Table 4. The average prediction accuracy score of the CNN model is $98 \%$ whereas the average prediction accuracy score for LSTM is $96 \%$ and RF is $80 \%$. CNN achieved the highest sensitivity and specificity among all techniques. The CNN and LSTM architectures outperformed the RF classifier for all k-mer sizes (CNN model: Sn97\%, Sp-99\% MCC-97\%; LSTM model: Sn-92\%, Sp-97\%, MCC-94\%; RF: Sn-58\%, Sp-87\%, MCC-65\%). For multiclass classification of sequences into promoter groups, the performance of RF in terms of accuracy and sensitivity has decreased with an increase in k-mer size. LSTM achieved the highest sensitivity and specificity at k-mer 4. The deep learning techniques CNN and LSTM outperformed RF (Fig. 4a), whereas change in the k-mer size does not show a significant difference in the prediction performance (Fig. 4b).

\section{Discussion}

A common practice in DNA sequence analysis is to use a set of 'background' sequences as negative controls for evaluation of the false-positive rates of gene recognition techniques for the detection of cis-regulatory elements. Generally, in the computational analysis of gene sequences, a set of non-target sequences extracted from the same genome is used as a background for evaluation purposes (Rani, Bhavani, and Bapi 2007). However, randomly selecting a nonpromoter region from the same genome as a negative dataset may have its own limitations. Such a model can easily find basic features to separate two classes but not the less obvious ones. Another approach to create a background dataset is shuffling of the nucleotide sequences of a fraction of positive dataset sequences. In our analysis, we found that such a synthetic background dataset of shuffled sequences resulted in models with high sensitivity and specificity of classification.

We used frequency-based tokenization of k-mers instead of one-hot encoding (Giosue and Gangi 2017; Nguyen et al. 2016; Umarov and Solovyev 2017) for feature encoding. In one-hot encoding each input sequence is thought as a matrix of 0 's and 1 's with the size $4^{\mathrm{k}} \mathrm{x}$ L, where $\mathrm{k}$ is the length of $\mathrm{k}$-mer and $\mathrm{L}$ is the length of the sequence. This input to embedding layer is highly sparse. The input dimension increases with an increase in k-mer which will automatically results in high computational processing time. Also, the one-hot encoded matrix representation of a sequence cannot capture the significance of the number of times the subsequence or motifs is going to occur. The advantage of frequency-based tokenization over one-hot encoding is that it gives a shorter input dimension to the AI model and can save training time significantly as compared to the one-hot encoding. Therefore, one-hot encoding may produce comparable results 
329

330

331

332

333

334

335

336

337

338

339

340

341

342

343

344

345

346

347

348

349

350

351

352

353

354

355

356

357

358

359

360

361

362

363

364

365

366

in terms of accuracy, however computational configuration required for implementation of one hot encoding for higher k-mer sizes may go beyond the computational support available with most of the researchers.

Optimization of parameters is crucial for sequence predictor construction. During the classification of sequences into a promoter and non-promoter categories, an empirically identified tuneable parameter was k-mer size along with the configuration of network architecture. With the change in k-mer size, the resulting feature vectors size also changed, affecting predictive performance of the models. We studied the effect of 2-mer, 4-mer and 8-mer on the prediction performance of CNN, LSTM and RF on distinct organisms. As mentioned earlier, the performance of the LSTM model improved with an increase in k-mer size. We also tried k-mer sizes of 12 and 16 for each of the models. However, this increased the number of training parameters exponentially resulting in a "resource exhaustion problem" due to the consumption of large amounts of memory. Further, study on the combination of featureextraction, feature encoding and the model architecture parameters is needed which can yield improvements in the prediction performance and help to reduce the execution time of the program.

We used four performance evaluation parameters Accuracy, Sensitivity (recall), Specificity and MCC. Accuracy is an average prediction performance on sample datasets. Still, accuracy alone cannot be an accurate measure to evaluate an ML model. Therefore, sensitivity and specificity are used for measuring the fraction of true positives and true negatives that are correctly predicted. With CNN and LSTM, deep learning models we have achieved both high sensitivity and specificity in all organisms.

The results show that the performance of CNN is better than LSTM and RF for all distinct organisms. The CNN models have reduced the number of false-positive and false-negative predictions and achieved high accuracy in both binary and multiclass classification. It demonstrates the ability of $\mathrm{CNN}$ to identify and extract abstract complex functional features with least pre-processing. In the case of cross-species evaluation, the performance of CNN is better than RF. However, the performance of both models is low, as promoters from the different species have different sequence structures, composition, and regulatory mechanisms. However, considering the differences in the type of data and data size, the differences observed in accuracy, sensitivity, specificity and MCC may not necessarily reflect on the model developed.

\section{Conclusions}

The primary aim of this work is to efficiently discriminate sequences into a promoter and nonpromoter sequences with a high true positive rate and true negative rate along with higher accuracy. We have proposed and demonstrated three specific improvements to the traditional methods for developing the generic and robust framework for classification tasks in the genomic domain. For our analysis, instead of using randomly selected non-promoter region we have 
367

368

369

370

371

372

373

374

375

376

377

378

379

380

381

382

383

384

385

386

387

388

389

390

391

392

393

394

395

396

397

398

399

400

401

402

utilized shuffled synthetic promoter sequences as a negative dataset to achieve necessary heterogeneity and robustness. A set of random sequences from human genome were used to test the efficiency of the models. For pre-processing of data, we have used k-mer based subsampling and frequency-based tokenization of sequences for feature extraction and vector representation respectively ensuring the reduction in training time. The deep learning techniques, namely, CNN and LSTM are employed for the classification of sequences into promoters and non-promoter categories which is important to interpret the underlying working of gene regulation. These methods are independent of the identification of any elements such as TATA-box, GC-box, CpG islands and sequence alignment methods for promoter prediction which are traditionally employed for this task. Using CNN, we achieved both high sensitivity and specificity while achieving higher accuracy on such a huge dataset. Results show a superiority of the CNN architecture over LSTM and RF in the binary and multispecies sequence classification. The effect of k-mer size on the model, both in terms of performance and training time is also extensively demonstrated. The proposed improvements and the CNN based approach are extremely generic and can be utilised to identify other elements of gene sequences and to meet the requirements of molecular biologists.

\section{Data availability}

Putative upstream regulatory regions used for this study, list of tools, and code snippet for reshuffling of sequences, k-merization of sequences along with CNN and LSTM architecture and model plot can be found at https://github.com/nikitabhandari-dl/Dataset

\section{Acknowledgment}

This work has been supported by the Scheme for Promotion of Academic and Research Collaboration (SPARC) 2018-19, MHRD (project no. P104). NB was supported by the Junior Research Fellowship Award 2018 by Symbiosis International Deemed University, India.

\section{Authors' Contribution}

NB performed the experiments, statistical analysis. NB and SK interpreted the results and wrote the manuscript. SK, RW, and KK outlined the research proposal. RW and KK reviewed the manuscript and inspired the overall analysis.

\section{References}

Anwar, Firoz, Syed Murtuza Baker, Taskeed Jabid, Md Mehedi Hasan, Mohammad Shoyaib, Haseena Khan, and Ray Walshe. 2008. "Pol II Promoter Prediction Using Characteristic 4Mer Motifs: A Machine Learning Approach.” BMC Bioinformatics 9: 1-8.

Bailey, Timothy L., Mikael Boden, Fabian A. Buske, Martin Frith, Charles E. Grant, Luca Clementi, Jingyuan Ren, Wilfred W. Li, and William S. Noble. 2009. "MEME Suite: Tools for Motif Discovery and Searching." Nucleic Acids Research 37(SUPPL. 2): 202-8. 
403

404

405

406

407

408

409

410

411

412

413

414

415

416

417

418

419

420

421

422

423

424

425

426

427

428

429

430

431

432

433

434

435

436

437

438

439

440

441

442

Blanchette, Mathieu, and Martin Tompa. 2003. "FootPrinter: A Program Designed for Phylogenetic Footprinting." Nucleic Acids Research 31(13): 3840-42.

Breiman, Leo. 2001. "Random Forests.” Machine Learning: 5-32. https://perun.pmf.uns.ac.rs/radovanovic/dmsem/cd/install/Weka/doc/classifierspapers/trees/RandomForest/Breiman2001-ML45.pdf.

Caballero, Juan, Arian F.A. Smit, Leroy Hood, and Gustavo Glusman. 2014. "Realistic Artificial DNA Sequences as Negative Controls for Computational Genomics." Nucleic Acids Research 42(12): 1-13.

Cheng, Feixiong, Weiqiang Lu, Chuang Liu, Jiansong Fang, Yuan Hou, Diane E. Handy, Ruisheng Wang, Yuzheng Zhao, Yi Yang, Jin Huang, David E. Hill, Marc Vidal, Charis Eng, and Joseph Loscalzo. 2019. "A Genome-Wide Positioning Systems Network Algorithm for in Silico Drug Repurposing." Nature Communications 10(1). http://dx.doi.org/10.1038/s41467-019-10744-6.

Chor, Benny, David Horn, Nick Goldman, Yaron Levy, and Tim Massingham. 2009. "Genomic DNA K-Mer Spectra: Models and Modalities.” Genome Biology 10(10).

Chowdhury, Biswanath, and Gautam Garai. 2017. “A Review on Multiple Sequence Alignment from the Perspective of Genetic Algorithm.” Genomics 109(5-6): 419-31. https://doi.org/10.1016/j.ygeno.2017.06.007.

Collobert, Ronan, and Jason Weston. 2008. "A Unified Architecture for Natural Language Processing: Deep Neural Networks with Multitask Learning.” Proceedings of the 25th International Conference on Machine Learning: 160-67.

Davuluri, Ramana V. 2003. "Application of FirstEF to Find Promoters and First Exons in the Human Genome." Current Protocols in Bioinformatics 1(1): 4.7.1-4.7.10.

Díaz-Uriarte, Ramón, and Sara Alvarez de Andrés. 2006. "Gene Selection and Classification of Microarray Data Using Random Forest." BMC bioinformatics 7: 3. http://www.ncbi.nlm.nih.gov/pubmed/16398926\%0Ahttp://www.pubmedcentral.nih.gov/art iclerender.fcgi?artid=PMC1363357.

Down, Thomas A., and Tim J.P. Hubbard. 2002. "Computational Detection and Location of Transcription Start Sites in Mammalian Genomic DNA.” Genome Research 12(3): 458-61.

Giosue, Bosco, and Mattia ANtonio Di Gangi. 2017. “Deep Learning Architectures for DNA Sequence Classification.” 10147: 249-59. https://link.springer.com/chapter/10.1007/978-3319-52962-2_14.

Gordon, Leo, Alexey Ya Chervonenkis, Alex J. Gammerman, Ilham A. Shahmuradov, and Victor V. Solovyev. 2003. "Sequence Alignment Kernel for Recognition of Promoter Regions." Bioinformatics 19(15): 1964-71.

Haeussler, Maximilian, Ann S. Zweig, Cath Tyner, Matthew L. Speir, Kate R. Rosenbloom, Brian J. Raney, Christopher M. Lee, Brian T. Lee, Angie S. Hinrichs, Jairo Navarro Gonzalez, David Gibson, Mark Diekhans, Hiram Clawson, Jonathan Casper, Galt P. Barber, David Haussler, Robert M. Kuhn, and W. James Kent. 2019. "The UCSC Genome Browser Database: 2019 Update.” Nucleic Acids Research 47(D1): D853-58. 
443

444

445

446

447

448

449

450

451

452

453

454

455

456

457

458

459

460

461

462

463

464

465

466

467

468

469

470

471

472

473

474

475

476

477

478

479

480

Hochreiter, Sepp, and Jürgen Schmidhuber. 1997. "LSTM Can Solve Hard Long Time Lag Problems." Advances in Neural Information Processing Systems: 473-79.

Ioshikhes, Ilya P., and Michael Q. Zhang. 2000. "Large-Scale Human Promoter Mapping Using CpG Islands." Nature Genetics 26(1): 61-63.

Kalkatawi, Manal, Arturo Magana-Mora, Boris Jankovic, and Vladimir B. Bajic. 2019. "DeepGSR: An Optimized Deep-Learning Structure for the Recognition of Genomic Signals and Regions." Bioinformatics 35(7): 1125-32.

Karthik, S, and M Sudha. 2018. "A Survey on Machine Learning Approaches in Gene Expression Classification in Modelling Computational Diagnostic System for Complex Diseases." (2): 182-91.

Knudsen, Steen. 1999. "Promoter2.0: For the Recognition of PolII Promoter Sequences." Bioinformatics 15(5): 356-61.

Lai, Hong Yan, Zhao Yue Zhang, Zhen Dong Su, Wei Su, Hui Ding, Wei Chen, and Hao Lin. 2019. "IProEP: A Computational Predictor for Predicting Promoter." Molecular Therapy Nucleic Acids 17(September): 337-46. https://doi.org/10.1016/j.omtn.2019.05.028.

Larrañaga, Pedro, Borja Calvo, Roberto Santana, Concha Bielza, Josu Galdiano, Iñaki Inza, José A. Lozano, Rubén Armañanzas, Guzmán Santafé, Aritz Pérez, and Victor Robles. 2006. "Machine Learning in Bioinformatics." Briefings in Bioinformatics 7(1): 86-112.

LeCun, Yann, Léon Bottou, Yoshua Bengio, and Patrick Haffner. 1998. "Gradient-Based Learning Applied to Document Recognition.” Proceedings of the IEEE 86(11): 2278-2323.

Li, Rudong, Yin Wang, Hui Cheng, Gang Liu, Tao Cheng, Yunlong Liu, and Lei Liu. 2017. "System Modeling Reveals the Molecular Mechanisms of HSC Cell Cycle Alteration Mediated by Maff and Egr3 under Leukemia." BMC Systems Biology 11(Suppl 5).

Li, Yifeng, Chih Yu Chen, and Wyeth W. Wasserman. 2016. "Deep Feature Selection: Theory and Application to Identify Enhancers and Promoters." Journal of Computational Biology 23(5): 322-36.

Libbrecht, Maxwell W, and William Stafford Noble. 2015. "Machine Learning in Genetics and Genomics." Nat Rev Genet 16(6): 321-32.

Liu, Rongxiang, and David J. States. 2002. "Consensus Promoter Identification in the Human Genome Utilizing Expressed Gene Markers and Gene Modeling." Genome Research 12(3): $462-69$.

Loots, G. G., I. Ovcharenko, L. Pachter, I. Dubchak, and E. M. Rubin. 2002. "RVista for Comparative Sequence-Based Discovery of Functional Transcription Factor Binding Sites." Genome Research 12(5): 832-39.

Manogaran, Gunasekaran, V. Vijayakumar, R. Varatharajan, Priyan Malarvizhi Kumar, Revathi Sundarasekar, and Ching Hsien Hsu. 2018. "Machine Learning Based Big Data Processing Framework for Cancer Diagnosis Using Hidden Markov Model and GM Clustering." Wireless Personal Communications 102(3): 2099-2116. 
481

482

483

484

485

486

487

488

489

490

491

492

493

494

495

496

497

498

499

500

501

502

503

504

505

506

507

508

509

510

511

512

513

514

515

516

517

518

Mathur, Meghna. 2013. "Multiple Sequence Alignment Using MATLAB.” International Journal of Information and Computation Technology 3(6): 497-504. http://www.

Matthews, B. W. 1975. "Comparison of the Predicted and Observed Secondary Structure of T4 Phage Lysozyme.” BBA - Protein Structure 405(2): 442-51.

Nguyen, Ngoc Giang, Vu Anh Tran, Duc Luu Ngo, Dau Phan, Favorisen Rosyking Lumbanraja, Mohammad Reza Faisal, Bahriddin Abapihi, Mamoru Kubo, and Kenji Satou. 2016. "DNA Sequence Classification by Convolutional Neural Network." Journal of Biomedical Science and Engineering 09(05): 280-86.

Ohler, Uwe. 2000. "Promoter Prediction on a Genomic Scale - The Adh Experience." Genome Research 10(4): 539-42.

Oubounyt, Mhaned, Zakaria Louadi, Hilal Tayara, and Kil To Chong. 2019. "Deepromoter: Robust Promoter Predictor Using Deep Learning." Frontiers in Genetics 10(APR): 1-9.

Pan, Xiaoyong, and Junchi Yan. 2017. "Attention Based Convolutional Neural Network for Predicting RNA-Protein Binding Sites.” (Nips): 8-11. http://arxiv.org/abs/1712.02270.

Pedregosa, Fabian, Gael Varoquaux, Alexandre Gramfort, Vincent Michel, Bertrand Thirion, Olivier Grisel, Mathieu Blondel, Peter Prettenhofer, Ron Weiss, Vincent Dubourg, Jake Vanderplas, Alexandre Passos, David Cournapeau, Matthieu Brucher, Matthieu Perrot, and Édouard. Duchesnay. 2011. "Scikit-Learn: Machine Learning in Python.” Journal of Machine Learning Research 12(January): 2825-30.

Ponger, Loïc, and Dominique Mouchiroud. 2002. “CpGProD: Identifying CpG Islands Associated with Transcription Start Sites in Large Genomic Mammalian Sequences." Bioinformatics 18(4): 631-33.

Rahman, Md Siddiqur, Usma Aktar, Md Rafsan Jani, and Swakkhar Shatabda. 2019. "IPromoterFSEn: Identification of Bacterial $\Sigma 70$ Promoter Sequences Using Feature Subspace Based Ensemble Classifier." Genomics 111(5): 1160-66.

Rani, T. Sobha, S. Durga Bhavani, and Raju S. Bapi. 2007. “Analysis of E.Coli Promoter Recognition Problem in Dinucleotide Feature Space." Bioinformatics 23(5): 582-88.

Sandelin, Albin, Wyeth W. Wasserman, and Boris Lenhard. 2004. "ConSite: Web-Based Prediction of Regulatory Elements Using Cross-Species Comparison.” Nucleic Acids Research 32(WEB SERVER ISS.): 249-52.

Singla-Pareek, SNEH LATA, M K Reddy, and SUDHIR K Sopory. 2001. "Transgenic Approach towards Developing Abiotic Stress Tolerance in Plants." proceeding-Indian national science academy part B 67(5): 265-84.

Skaik, Younis. 2008. "Understanding and Using Sensitivity, Specificity and Predictive Values." Indian Journal of Ophthalmology 56(4): 341.

Solovyev, Victor V., and I. A. Shahmuradov. 2003. "PromH: Promoters Identification Using Orthologous Genomic Sequences." Nucleic Acids Research 31(13): 3540-45.

Tabl, Ashraf Abou, Abedalrhman Alkhateeb, Waguih Elmaraghy, and Luis Rueda. 2019. “A 
519 Machine Learning Approach for Identifying Gene Biomarkers Guiding the Treatment of 520 Breast Cancer." 10(March): 1-13.

521 Le Thi, Hoai An, Van Vinh Nguyen, and Samir Ouchani. 2008. "Gene Selection for Cancer 522 Classification Using DCA." Lecture Notes in Computer Science (including subseries $523 \quad$ Lecture Notes in Artificial Intelligence and Lecture Notes in Bioinformatics) 5139 LNAI:

$524 \quad 62-72$.

525 Umarov, Ramzan Kh, and Victor V. Solovyev. 2017. "Recognition of Prokaryotic and Eukaryotic Promoters Using Convolutional Deep Learning Neural Networks.” PLoS ONE

Wang, Jason T L, Steve Rozen, Bruce A Shapiro, Dennis Shasha, Zhiyuan Wang, Maisheng Yin, and Wang E T Al. 1999. “Techniques Sequence.” 6(2): 209-18.

Wang, Liangjiang, Mary Qu Yang, and Jack Y. Yang. 2009. "Prediction of DNA-Binding Residues from Protein Sequence Information Using Random Forests." BMC Genomics 10(SUPPL. 1): 1-9.

Yang, Pengyi, Yee Hwa Yang, Bing B. Zhou, and Albert Y. Zomaya. 2010. "A Review of Ensemble Methods in Bioinformatics." Current Bioinformatics 5(4): 296-308. 
Figure 1

Steps for classification of DNA sequence: feature extraction, feature encoding and classification

The first step is to input Raw sequences for feature extraction and feature encoding using Kmerization and frequency-based tokenization techniques, respectively. Then the tokenized sequence fed to ML or DL model for further analysis. For deep learning, CNN and LSTM are used, whereas for machine learning RF is used.

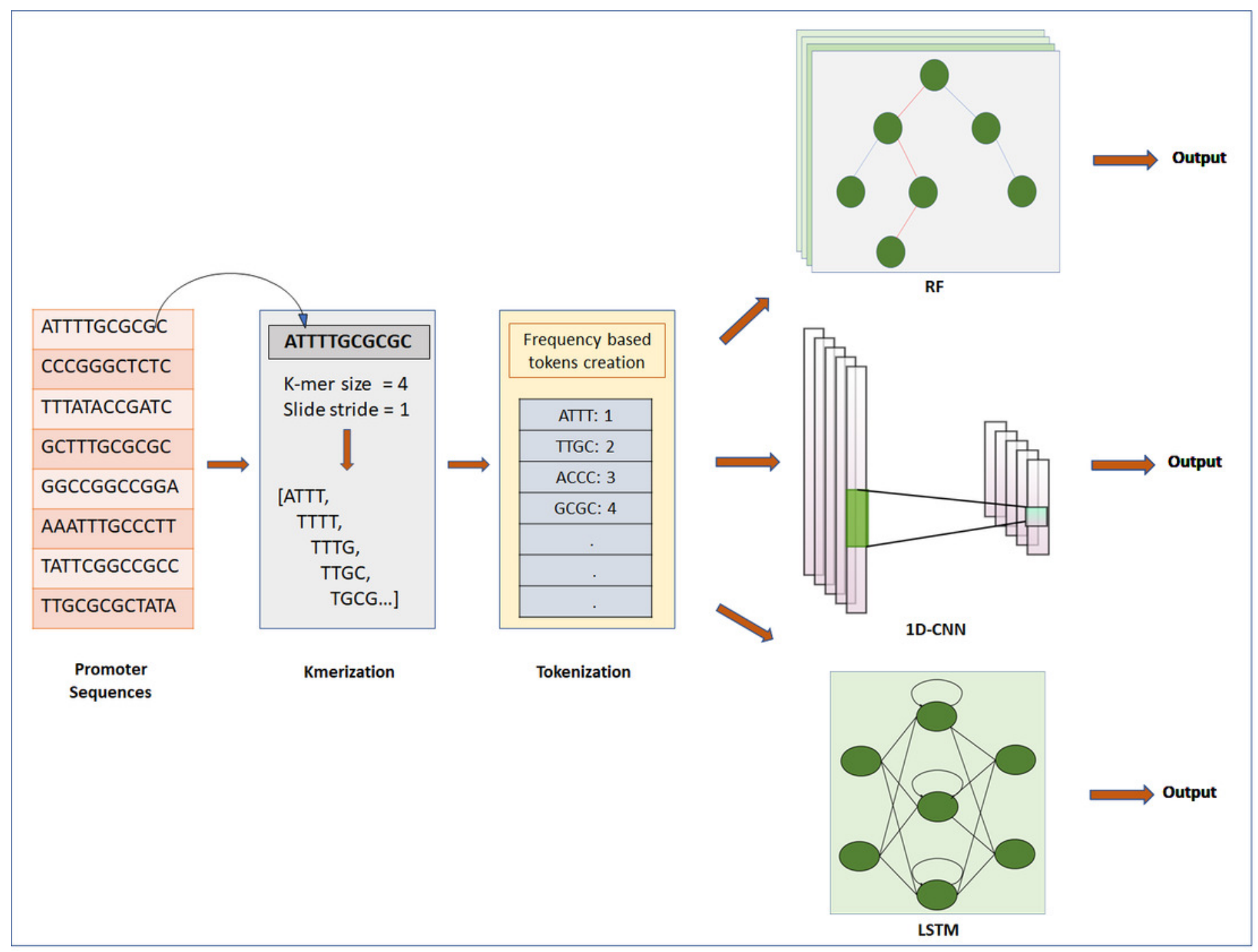


Figure 2

Performance analysis of CNN, LSTM, and RF for binary classification. Three distinct organisms (yeast, Arabidopsis thaliana, human) were used

(a), (b) and (c): The accuracy performance of CNN, LSTM, and RF for various k-mer sizes. A significant difference in the performance of $D L$ and $M L$ techniques for each k-mer size was observed. (d), (e) and (f): The accuracy performance of CNN, LSTM, and RF on each species. A significant difference in the performance of $D L$ and $M L$ techniques for each organism was observed. (* statistically significant, ** statistically more significant, n.s. non-significant)

(a)

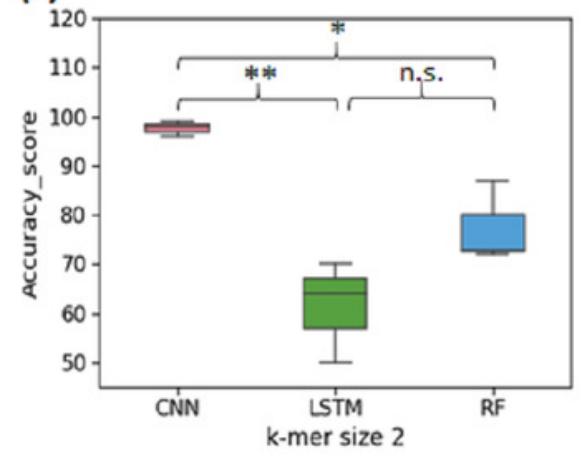

(d)

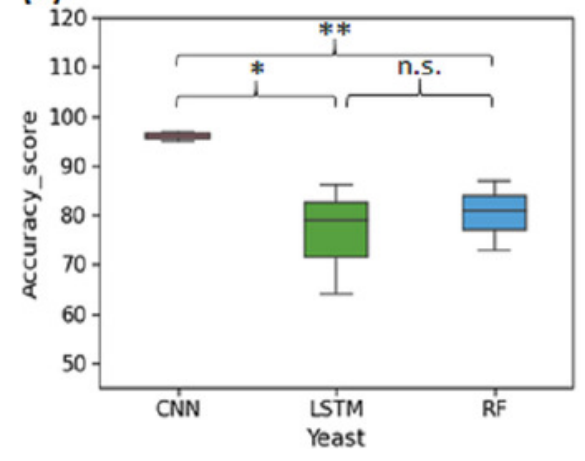

(b)

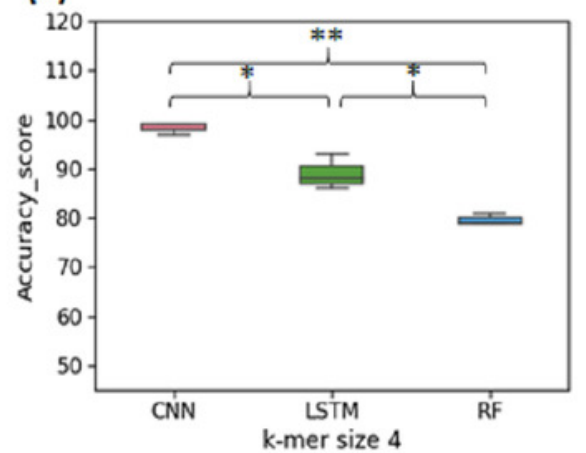

(e)

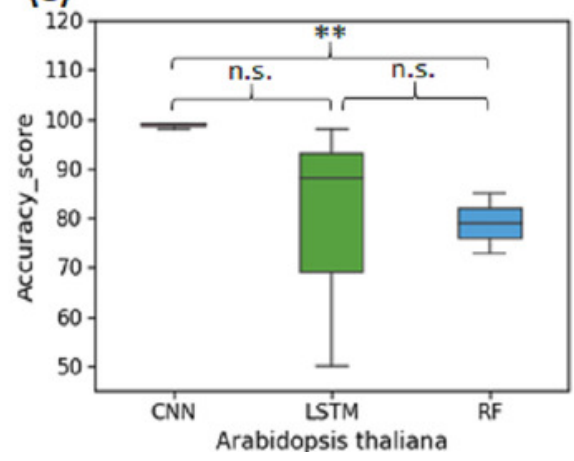

(c)

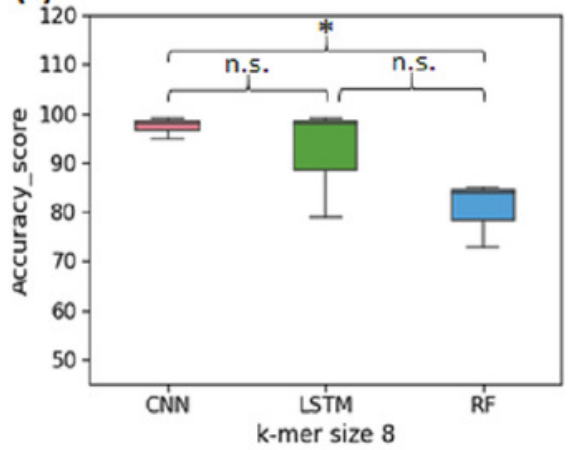

(f)

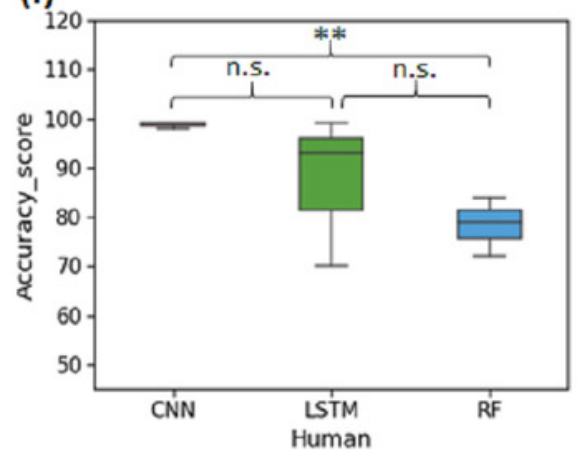


Figure 3

Performance analysis of CNN and RF for cross-species evaluation

(a)

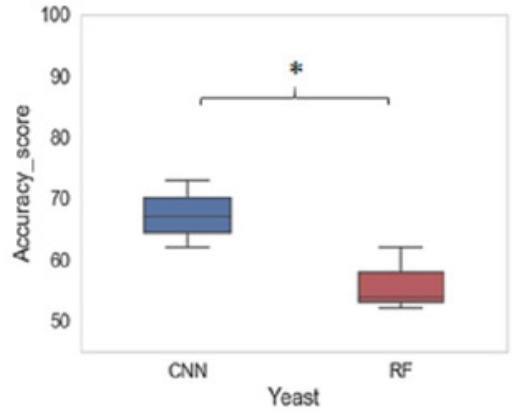

(d)

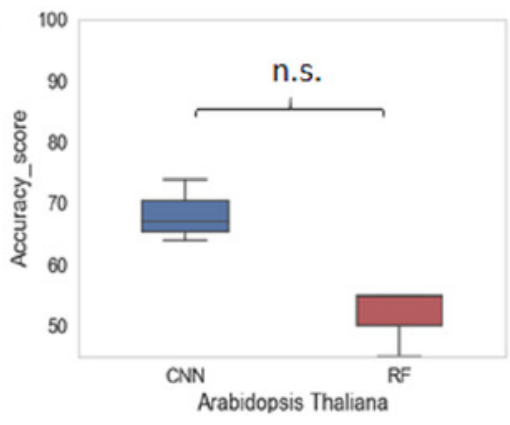

(b)

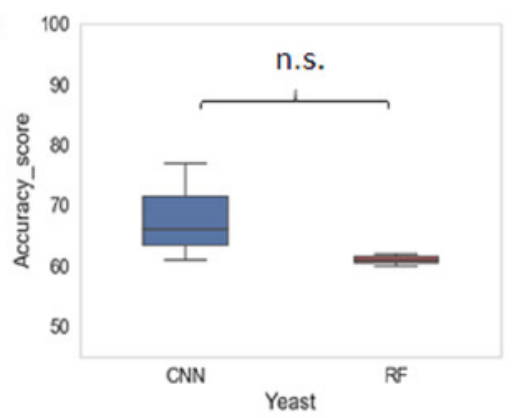

(e)

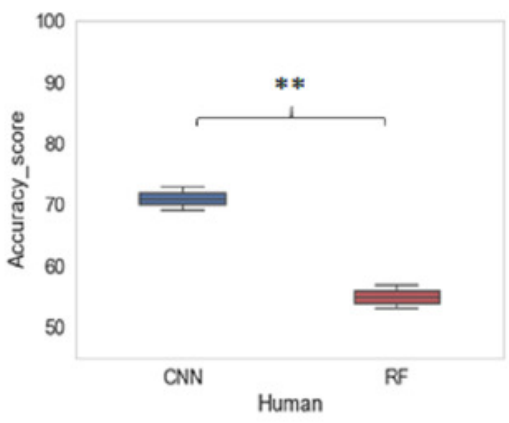

(c)

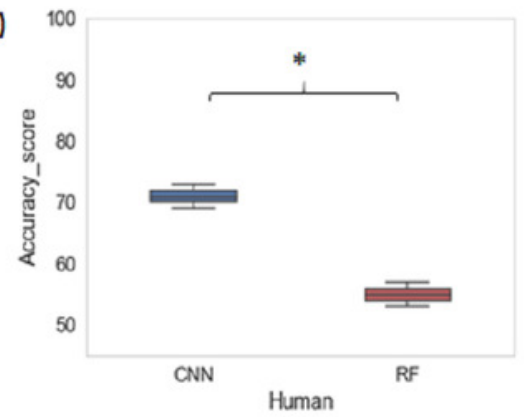

(f)

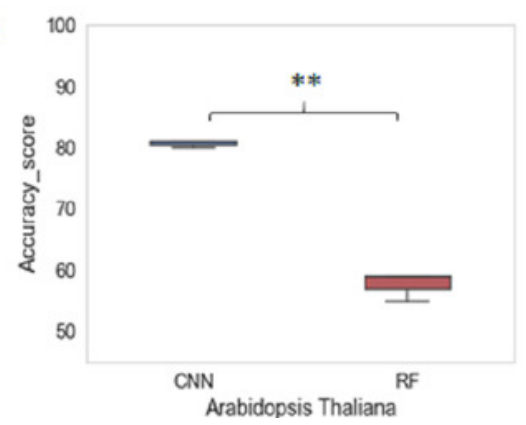


Figure 4

Performance analysis of CNN, LSTM, and RF for multi-species promoter sequence classification

(a)

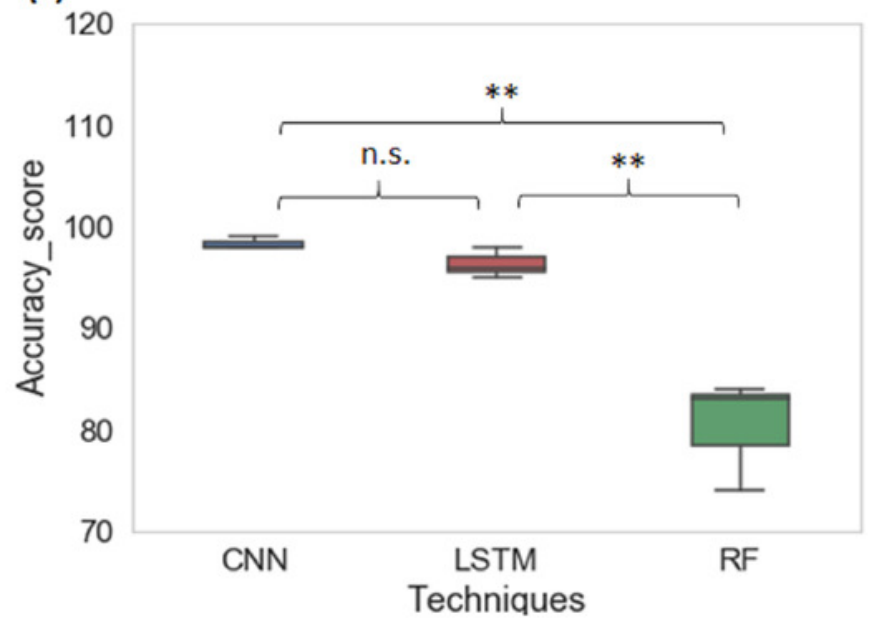

(b)

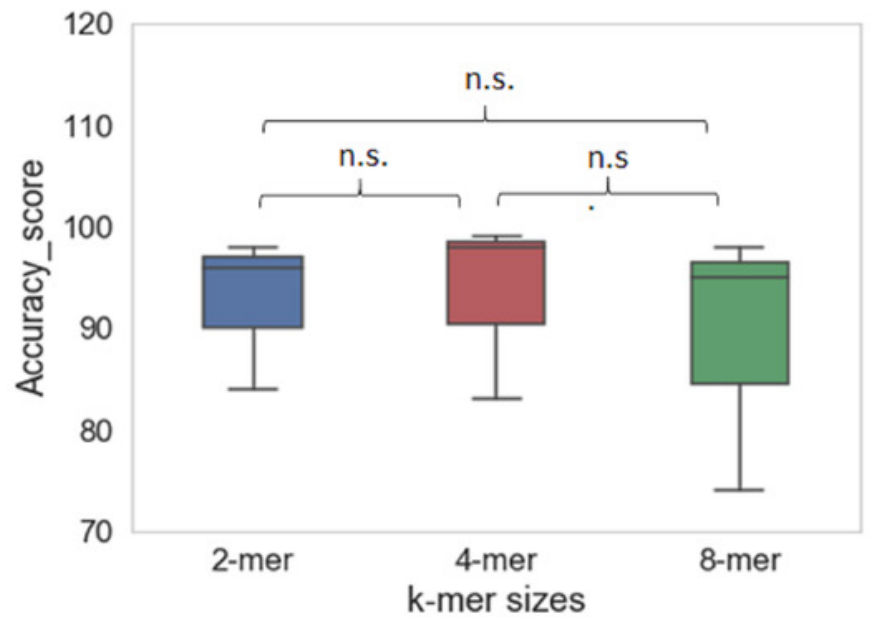




\section{Table $\mathbf{1}$ (on next page)}

Input dimensions and training time for corresponding encoding techniques and k-mer sizes. 
3 Table 1:

4 Input dimensions and training time for corresponding encoding techniques and k-mer

5 sizes.

6

\begin{tabular}{|c|c|c|c|c|c|c|}
\hline Techniques & $\begin{array}{c}\text { Training } \\
\text { time (min) }\end{array}$ & $\begin{array}{c}\text { Input Vector } \\
\text { size }\end{array}$ & Acc & Sn & Sp & MCC \\
\hline & \multicolumn{6}{|c|}{ 1-mer } \\
\hline One Hot Encoding & 28 & $4 \times 1000$ & 0.95 & 0.98 & 0.90 & 0.90 \\
\hline \multirow[t]{2}{*}{$\begin{array}{c}\text { Frequency Based } \\
\text { Tokenization }\end{array}$} & 14 & $1 \times 1000$ & 0.97 & 0.98 & 0.99 & 0.97 \\
\hline & \multicolumn{6}{|c|}{ 2-mer } \\
\hline One Hot Encoding & 240 & $16 \times 999$ & 0.96 & 0.97 & 0.95 & 0.93 \\
\hline $\begin{array}{c}\text { Frequency Based } \\
\text { Tokenization }\end{array}$ & 14.3 & $1 \times 999$ & 0.96 & 0.98 & 0.93 & 0.89 \\
\hline
\end{tabular}

7

8

9 


\section{Table 2 (on next page)}

Performance of CNN, LSTM and RF models for binary classification using different statistical measures 
1 Table 2:

2 Performance of CNN, LSTM and RF models for binary classification using different

3 statistical measures.

\begin{tabular}{|c|c|c|c|c|c|c|c|c|c|c|c|c|}
\hline \multirow{2}{*}{ Methods } & \multicolumn{4}{|c|}{ Yeast } & \multicolumn{4}{|c|}{ Arabidopsis thaliana } & \multicolumn{4}{|c|}{ Human } \\
\hline & Acc & Sn & Sp & MCC & Acc & Sn & Sp & $\mathrm{MCC}$ & Acc & Sn & Sp & MCC \\
\hline & \multicolumn{12}{|c|}{ 2-mer } \\
\hline CNN & 0.96 & 0.98 & 0.93 & 0.89 & 0.98 & 0.97 & 0.99 & 0.95 & 0.99 & 0.99 & 0.99 & 0.99 \\
\hline LSTM & 0.64 & 0.56 & 0.71 & 0.27 & 0.50 & 0.50 & 0.50 & 0.01 & 0.70 & 0.69 & 0.74 & 0.49 \\
\hline \multirow[t]{2}{*}{ RF } & 0.87 & 0.84 & 0.90 & 0.74 & 0.73 & 0.76 & 0.70 & 0.46 & 0.72 & 0.76 & 0.68 & 0.45 \\
\hline & \multicolumn{12}{|c|}{ 4-mer } \\
\hline CNN & 0.97 & 0.99 & 0.95 & 0.91 & 0.99 & 1.00 & 0.99 & 0.98 & 0.99 & 1.00 & 0.99 & 0.99 \\
\hline LSTM & 0.86 & 0.91 & 0.82 & 0.73 & 0.88 & 0.88 & 0.90 & 0.78 & 0.93 & 0.94 & 0.95 & 0.89 \\
\hline \multirow[t]{2}{*}{ RF } & 0.81 & 0.74 & 0.88 & 0.62 & 0.79 & 0.80 & 0.79 & 0.59 & 0.79 & 0.80 & 0.80 & 0.54 \\
\hline & \multicolumn{12}{|c|}{ 8-mer } \\
\hline CNN & 0.95 & 0.95 & 0.96 & 0.91 & 0.99 & 0.99 & 0.99 & 0.98 & 0.98 & 0.99 & 0.98 & 0.98 \\
\hline LSTM & 0.79 & 0.82 & 0.77 & 0.58 & 0.98 & 0.97 & 0.98 & 0.97 & 0.99 & 0.99 & 0.99 & 0.98 \\
\hline RF & 0.73 & 0.66 & 0.81 & 0.47 & 0.85 & 0.82 & 0.88 & 0.69 & 0.84 & 0.81 & 0.87 & 0.69 \\
\hline
\end{tabular}

4 


\section{Table 3 (on next page)}

Performance of CNN, LSTM and RF models for binary classification when random sequences from human genome are used as negative class data with test data 
1 Table 3:

2 Performance of CNN, LSTM and RF models for binary classification when random

3 sequences from human genome are used as negative class data with test data.

4

\begin{tabular}{|c|c|c|c|c|}
\hline \multirow{2}{*}{ Methods } & \multicolumn{4}{|c|}{ Human } \\
\cline { 2 - 5 } & Acc & Se & Sp & MCC \\
\hline & \multicolumn{4}{|c|}{ 2-mer } \\
\hline CNN & 0.9 & 0.85 & 0.96 & 0.84 \\
\hline LSTM & 0.56 & 0.09 & 0.96 & 0.01 \\
\hline RF & 0.75 & 0.77 & 0.72 & 0.49 \\
\hline & \multicolumn{5}{|c|}{ 4-mer } \\
\hline CNN & 0.91 & 0.88 & 0.99 & 0.85 \\
\hline LSTM & 0.89 & 0.82 & 0.96 & 0.79 \\
\hline RF & 0.79 & 0.77 & 0.81 & 0.58 \\
\hline & \multicolumn{4}{|c|}{ 8-mer } \\
\hline CNN & 0.91 & 0.85 & 0.98 & 0.83 \\
\hline LSTM & 0.91 & 0.84 & 0.99 & 0.84 \\
\hline RF & 0.82 & 0.77 & 0.86 & 0.64 \\
\hline
\end{tabular}

5

6 


\section{Table 4 (on next page)}

Performance of CNN, LSTM and RF models for multi-species classification using different statistical measures. 
1 Table 4:

2 Performance of CNN, LSTM and RF models for multi-species classification using different 3 statistical measures.

4

\begin{tabular}{|c|c|c|c|c|}
\hline \multirow{2}{*}{ Methods } & Acc & Sn & Sp & MCC \\
\cline { 2 - 5 } & \multicolumn{4}{|c|}{ 2-mer } \\
\hline CNN & 0.98 & 0.97 & 0.99 & 0.97 \\
\hline LSTM & 0.96 & 0.93 & 0.94 & 0.93 \\
\hline RF & 0.84 & 0.61 & 0.89 & 0.73 \\
\hline & \multicolumn{5}{|c|}{ 4-mer } \\
\hline CNN & 0.99 & 0.98 & 0.99 & 0.98 \\
\hline LSTM & 0.98 & 0.96 & 0.99 & 0.96 \\
\hline RF & 0.83 & 0.60 & 0.89 & 0.70 \\
\hline & \multicolumn{5}{|c|}{8 -mer } \\
\hline CNN & 0.98 & 0.96 & 0.99 & 0.97 \\
\hline LSTM & 0.95 & 0.86 & 0.97 & 0.92 \\
\hline RF & 0.74 & 0.54 & 0.83 & 0.52 \\
\hline
\end{tabular}

5 